\title{
THE TRANSFER AND COMPACT LIE GROUPS
}

\author{
BY MARK FESHBACH
}

Communicated by Edgar H. Brown, Jr., November 1, 1976

1. Introduction. A map $\rho: X \rightarrow Y$ between two spaces induces a homomorphism $\rho^{*}: h(Y) \rightarrow h(X)$ between the cohomology groups of the spaces, where $h$ is an arbitrary cohomology theory. In certain situations a transfer homomorphism $\tau^{*}: h(X) \rightarrow h(Y)$ has been defined by Becker and Gottlieb, Dold and others. The compositions $\tau^{*} \circ \rho^{*}: h(Y) \rightarrow h(Y)$ and $\rho^{*} \circ \tau^{*}: h(X) \rightarrow h(X)$ are of considerable interest as they relate the cohomologies of $X$ and $Y$. The first type of composition is relatively easy to compute. The second is, in general, quite difficult.

Let $G$ be a compact Lie group with $H$ and $K$ arbitrary closed subgroups with associated $l$-universal classifying spaces $B G, B H, B K$. Let $\rho(H, G): B H \rightarrow$ $B G$ be the natural projection. Then transfers $T(H, G): h(B H) \rightarrow h(B G), T(K, G)$ : $h(B K) \rightarrow h(B G)$ are defined by Dold's definition where $T(H, G)=T_{\text {id }}^{B H}$ in Dold's notation [D]. The main theorem is a double coset type theorem which generalizes the classical double coset theorem for finite groups [C-E, p. 257]. It is proved for arbitrary compact Lie groups.

2. Main result. Let $K|G| H$ be the double coset space obtained as the orbit space of the left action of $K$ on $G / H$. This space breaks up into a finite disjoint union of orbit-type manifold components $\left\{M_{i}\right\}$. Let $g_{i} \in G$ be a representative of $M_{i}$. Let $\chi^{\#}\left(M_{i}\right)=\chi\left(\bar{M}_{i}\right)-\chi\left(\bar{M}_{i}-M_{i}\right)$ be the internal Euler characteristic of $M_{i}$. Then if $H^{g}=g \mathrm{Hg}^{-1}$ we have

Theorem 1 (Double Coset).

$$
\rho^{*}(K, G) \circ T(H, G)=\sum \chi^{\#}\left(M_{i}\right) T\left(H^{g_{i}} \cap K, K\right) \circ \rho^{*}\left(H^{g_{i}} \cap K, H^{g_{i}}\right) \circ C g_{i}
$$

where the sum is over the orbit-type manifold components of $K|G| H . C g: h(B H)$ $\rightarrow h\left(B H^{g}\right)$ is the cohomology isomorphism induced by the obvious map from $B H^{g}$ to $B H$.

This theorem holds where $G$ is a compact Lie group and $H$ and $K$ are arbitrary closed subgroups.

AMS (MOS) subject classifications (1970). Primary 55F40.

Key words and phrases. Transfer, classifying spaces, compact Lie group, double coset theorem.

${ }^{1}$ Based on a thesis written at Stanford University under the supervision of Professor G. Brumfiel. 
Theorem 1 simplifies in special cases, e.g. when it is known that $\chi^{\#}\left(M_{i}\right)=$ 0. In particular, the following easily proved result is often useful.

TheOREM 2. Assume $N_{G}(H) / H$ is not discrete where $N_{G}(H)$ is the normalizer of $H$ in $G$. Then $T(H, G)=0$.

3. Examples. In the case where $G$ is finite the double coset space $K|G| H$ is discrete. Since $\chi^{\#}(\mathrm{pt})=$.1 we recover the classical double coset theorem for finite groups.

Among many other corollaries to Theorem 1 we have

THEOREM 3. Let $G$ be a compact Lie group with $H$ any closed subgroup of $G$. Let $K$ be an arbitrary torus in $G$. Then

$$
\rho^{*}(K, G) \circ T(H, G)=\sum \chi^{\#}\left(M_{i}\right) \rho^{*}\left(K, H^{g_{i}}\right) \circ C g_{i}
$$

where the sum is over the manifold components of the fixed point set.

THEOREM 4. Let $G(n)=U(n), H(n)=\Sigma_{n} 2 U(1)$ the wreath product of the permutation group on $n$ letters $\Sigma_{n}$ and $U(1)$. Let $K(n)=U(n-1)$. Then the diagram

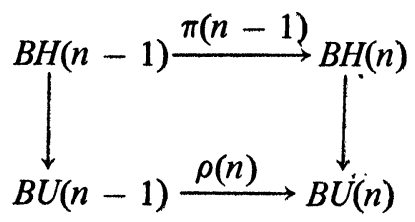

satisfies the following stability condition

$$
\rho^{*}(n) \circ T(H(n), U(n))=T(H(n-1), U(n-1)) \circ \pi^{*}(n-1) .
$$

A similar formula for the orthogonal groups appears in printed version of a talk by J. C. Becker [B] .

THEOREM 5. Let $G(n+m)=U(n+m), H(n+m)=\Sigma_{n+m} 2 U(1)$, $K(n, m)=U(n) \times U(m)$. Then the diagram

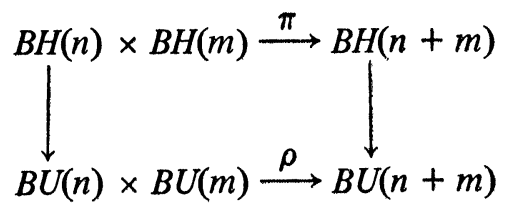

satisfies the following relation

$$
\text { ค*。 } T(H(n+m), U(n+m))=T(H(n) \times H(m), U(n) \times U(m)) \circ \pi^{*} .
$$

Both Theorems 4 and 5 are proven by using Theorem 2 to simplify the double coset formula to a single term.

4. Proof. The proof of Theorem 1 is geometric and involves constructing 
a $K$-equivariant deformation of the identity map of $G / H$ by appealing to the covering homotopy theorem of Palais [Br, p. 97]. Elementary properties of the transfer are then used. The proof with minor modifications applies to more general situations.

\section{REFERENCES}

[B] J. C. Becker, Characteristic classes and $K$-theory (talk).

[Br] G. E. Bredon, Introduction to compact transformation groups, Academic Press, New York, 1972.

[C-E] H. Cartan and S. Eilenberg, Homological algebra, Princeton Univ. Press, Princeton, N. J., 1956. MR 17, 1040.

[D] A. Dold, Transfert des points fixes d'une famille continue d'applications, C. R. Acad. Sci. Paris Sér. A 278 (1974), 1291-1293. MR 50 \#1231. 1976.

[F] Mark Feshbach, The transfer and compact Lie groups, Thesis, Stanford Univ.,

DEPARTMENT OF MATHEMATICS, NORTHWESTERN UNIVERSITY, EVANSTON, ILLINOIS 60201 